\title{
Disability and Depressive Symptoms in Later Life: The Stress-Buffering Role of Informal and Formal Support
}

\author{
Natalie Chan ${ }^{a}$ Kaarin J. Anstey ${ }^{a}$ Tim D. Windsor ${ }^{a}$ Mary A. Luszcz ${ }^{b}$

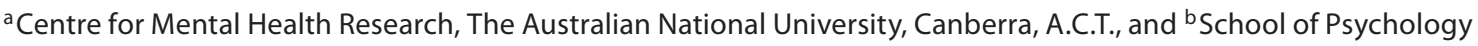 \\ and Centre for Ageing Studies, Flinders University, Adelaide, S.A., Australia
}

\section{Key Words}

Informal support · Formal support · Disability $\cdot$ Depressive symptoms $\cdot$ Home care

\begin{abstract}
Background: Despite the increasing utilization of formal services by older adults in many nations, there is a paucity of research that has examined the relationships between disability, formal support and depressive symptoms in later life. Objective: We investigated whether support received for daily activities, either from formal and/or informal sources, weakened the deleterious relationship between disability and depression symptoms in later life and whether these stress-buffering effects were stronger in later years of older adulthood. Methods: Participants were 1,359 communitydwelling older adults drawn from Wave 1 of the Australian Longitudinal Study of Ageing. Hierarchical multiple regression was used. Results: Individuals receiving support from (1) informal sources only and (2) both informal and formal sources had weaker relationships between disability and depressive symptoms, relative to those receiving no support. The interaction between informal and formal support and disability also revealed that for individuals with above average functional limitations, receipt of this support type was
\end{abstract}

associated with fewer depressive symptoms. However, for individuals with no functional limitations, receipt of both informal and formal support was not associated with depressive symptoms. The stress-buffer age variation hypothesis received no support. Conclusions: Findings suggest that receiving a combination of informal and formal support may be sufficient to offset the harmful association between disability and depressive symptoms in later life. In addition, findings further emphasized the importance of informal support in later life. In contrast, formal support in isolation may not be sufficient to confer a protective effect. Given the expected increase in utilization of formal services among older adults in the coming decades, it is essential that future research investigates the possible factors that underlie this null result.

Copyright $\odot 2010$ S. Karger AG, Basel

\section{Introduction}

Promoting healthy aging among community-dwelling older adults is a central component of aging policies in many nations. An important aspect of this involves ensuring that psychological health is maintained during the years lived with functional disability, which refers to

\section{KARGER}

๑) 2010 S. Karger AG, Basel

Fax +4161306 1234

E-Mail karger@karger.ch

www.karger.com
Accessible online at: www.karger.com/ger
Natalie Chan, BPsych(Hons)

Centre for Mental Health Research, Building 63

The Australian National University, Canberra, A.C.T. 0200 (Australia)

Tel. +61 26125 1448, Fax +61 261250733

E-Mail Natalie.Chan@anu.edu.au 
the extent to which physical pathology affects an individual's mobility [1]. To this end, much research has focused upon the role of informal enacted support (actual assistance received from individuals such as family and friends) [2]. In contrast, to our knowledge, no study has yet examined whether enacted support received from formal sources (i.e., organizations) may also protect psychological health against the increased risk of depression associated with functional disability. This is despite the fact that the popularity of these home care services has significantly increased over the past decade and that this growth is expected to steadily continue [3]. In this study, we investigate whether formal enacted support, received either in isolation or in addition to existing informal support, confers a protective effect indicated by a weaker negative association between disability and depressive symptoms among those receiving support. In addition, we examine the recently proposed hypothesis that the stress-buffering effect is especially evident in the later years of older adulthood [4].

\section{Functional Disability, Depressive Symptoms and Enacted Support}

Functional disability has been found to be related to a range of negative health outcomes including higher depressive symptoms [5-7], higher mortality risk [8] and lower life satisfaction [9]. Functional disability is thought to negatively impact psychological health by harming an individual's ability to manage daily social and instrumental activities, which are often essential to living independently and maintaining social relationships $[10,11]$. Although not inevitable, functional disability is increasingly common in later life [12]. Considering the prevalence of disabilities in older adulthood and their potential harmful effects, it is pertinent to investigate possible protective factors that may weaken the deleterious relationship between functional disability and psychological health to help maximize healthy aging.

As mentioned previously, the majority of research on this topic has focused upon the protective role of enacted support from informal sources. Three types of enacted support have been proposed: (1) emotional support, referring to the provision of care, warmth and understanding; (2) instrumental support, referring to practical assistance for daily activities, and (3) informational support, e.g. provision of knowledge and advice [13]. Typically, this research has been conducted within the stress-buffer paradigm [e.g. 14-16], which views functional disability as a chronic stressor that places continuous and ongoing demands upon the individual, and informal enacted sup- port as a protective coping mechanism that buffers the effects of the chronic stressor [11] by promoting more positive interpretations of adverse experiences and/or providing an individual with effective coping strategies $[17,18]$. In particular, it is argued that for the type of support to be protective, it is necessary that the support directly addresses the specific impact of the stressor [13]. Instrumental enacted support, whether it is received from informal or formal sources, aims to directly address limitations caused by disability. Given this, it is also possible that instrumental support from formal sources protects psychological health in later life against the negative effects of functional disability by reducing the magnitude of such effects upon an individual's ability to undertake daily instrumental and social activities.

\section{Stress-Buffer Age Variation Hypothesis}

Recently, there has been a suggestion in the literature that the stress-buffering effects of enacted support are stronger in the later years of older adulthood [4]. Reasons for this include: (1) that the relationships remaining in advanced old age (75 years and over) are likely to be particularly effective in reducing the effects of stressors according to socioemotional selectivity theory, the proposal that older individuals prefer relationships that assist with emotional regulation as they perceive time to be increasingly limited in later life [19], and (2) physical and cognitive changes in late life lead older adults to be more vulnerable to stress and that because of this, they will be more likely to draw upon external resources such as informal social support as a coping resource [4]. The stressbuffer age variation hypothesis has received empirical support from two cross-sectional studies that included life satisfaction as an outcome variable $[4,20]$. Research has yet to examine whether the protective effects of enacted support are also stronger in advanced old age with regard to depressive symptoms.

\section{Aims and Hypotheses}

The present study investigates whether (1) type of support received (informal only, formal only, or both informal and formal support) differentially moderates the relationship between disability and depressive symptoms as compared to the receipt of no support and (2) whether the strength of these stress-buffering effects varies with age. We hypothesize that all types of support will be associated with a weaker relationship between disability and depressive symptoms relative to those receiving no support and that this benefit will be greater at older ages. 
Table 1. Descriptive statistics for study variables by support type $(n=1,359)$

\begin{tabular}{|c|c|c|c|c|c|c|c|c|c|c|c|c|c|c|c|}
\hline \multirow[t]{2}{*}{ Characteristics } & \multirow[t]{2}{*}{ Range } & \multirow[t]{2}{*}{$\begin{array}{l}\text { Miss. }^{\mathrm{a}} \\
\%\end{array}$} & \multicolumn{3}{|c|}{$\begin{array}{l}\text { No support } \\
(\mathrm{n}=886)\end{array}$} & \multicolumn{3}{|c|}{$\begin{array}{l}\text { Informal only } \\
(\mathrm{n}=229)\end{array}$} & \multicolumn{3}{|c|}{$\begin{array}{l}\text { Formal only } \\
(\mathrm{n}=56)\end{array}$} & \multicolumn{3}{|c|}{$\begin{array}{l}\text { Both }^{\mathrm{a}} \\
(\mathrm{n}=118)\end{array}$} & \multirow[t]{2}{*}{$p\left(f, \chi^{2}\right)^{b}$} \\
\hline & & & $\%$ & M & SD & $\%$ & M & $\mathrm{SD}$ & $\%$ & M & $\mathrm{SD}$ & $\%$ & M & $\mathrm{SD}$ & \\
\hline \multicolumn{16}{|c|}{ Sociodemographic characteristics } \\
\hline Age & $70-103$ & 0 & & 77.70 & 5.74 & & $80.87^{\mathrm{c}}$ & 6.15 & & $80.54^{\mathrm{c}}$ & 5.94 & & $82.38^{\mathrm{c}}$ & 6.22 & 0.000 \\
\hline Female & & 0 & $28.6^{c}$ & & & $46.70^{c}$ & & & 42.90 & & & $52.10^{c}$ & & & 0.000 \\
\hline Income: USD $\leq 12,000$ & & 5.7 & $37.7^{\mathrm{c}}$ & & & 48.50 & & & 39.30 & & & $56.90^{c}$ & & & 0.000 \\
\hline Living alone & & 1.4 & $37.2^{\mathrm{c}}$ & & & 42.40 & & & 55.40 & & & $61.70^{c}$ & & & 0.000 \\
\hline \multicolumn{16}{|c|}{ Physical and cognitive health } \\
\hline Cog. imp. ${ }^{\mathrm{d}}$ & & 2.3 & $9.7^{\mathrm{c}}$ & & & $21.40^{c}$ & & & 7.10 & & & $22.30^{c}$ & & & 0.000 \\
\hline Chronic conds. $^{a}$ & $0-5$ & 0.1 & & 1.46 & 1.11 & & $1.89^{c}$ & 1.16 & & $1.89^{c}$ & 1.07 & & $1.98^{c}$ & 1.36 & 0.000 \\
\hline Medications & $0-5$ & 0.4 & & 2.45 & 1.71 & & $3.20^{c}$ & 1.60 & & $3.70^{c}$ & 1.39 & & $3.41^{\mathrm{c}}$ & 1.56 & 0.000 \\
\hline Func. lims. ${ }^{a}$ & $0-16.12$ & 4.9 & & 2.54 & 2.98 & & 6.39 & 4.34 & & 4.87 & 3.74 & & 8.29 & 4.69 & 0.000 \\
\hline \multicolumn{16}{|l|}{ Psychological health } \\
\hline Dep. symptoms ${ }^{\mathrm{a}}(\log )$ & $0-1.69$ & 4.9 & & 0.76 & 0.38 & & $0.92^{c}$ & 0.37 & & $0.93^{\mathrm{c}}$ & 0.34 & & $0.99^{c}$ & 0.32 & 0.000 \\
\hline
\end{tabular}

${ }^{\mathrm{a}}$ Miss. $=$ Missing data; Both $=$ both informal and formal support; Cog. imp. = cognitive impairment; Chronic conds. = chronic conditions; Func . lims. $=$ functional limitations; Dep. symptoms $=$ depressive symptoms.

${ }^{b}$ One-way ANOVA with post-hoc comparisons and Pearson $\chi^{2}$ analyses with adjusted standardized residuals were run to determine differences in means and proportions.
${ }^{c}$ For means - indicates value is significantly different as compared to the value of those who receive no support at $\mathrm{p}<0.01$. For percentages - indicates observed values are significantly different from expected values at $\mathrm{p}<0.01$.

${ }^{\mathrm{d}}$ Mini-Mental State Examination scaled score $\leq 23$.

\section{Methods}

\section{Covariates}

We controlled for a number of characteristics that are known to be related to depressive symptoms including sociodemographic characteristics, cognitive status, social networks and physical pathology. Further, variables that have been found to distinguish individuals who receive informal and/or formal support (co-resident status, social networks and functional limitations) were also included [21, 22].

\section{Participants}

The sample was drawn from Wave 1 of the Australian Longitudinal Study of Ageing (ALSA). The target population was all persons living in Adelaide, South Australia, aged 70 years and over on June 30, 1992 [for more details, see 23]. In brief, ALSA's sample was randomly selected from the South Australian Electoral Roll, which was stratified by gender and age into four groups, $70-74,75-79,80-84$ and 85 years and over. Response rate at Wave 1 was $55 \%$ with the total target sample comprising of 1,477 participants with 1,359 (92.0\% of the sample) residing in the community. The data collection method was a comprehensive face-toface structured home interview conducted by trained interviewers using the Computer-Assisted Personal Interviewing software [24]. Participants provided written consent and ethics approval was granted by the Clinical Investigations Committee of the Flinders Medical Centre in South Australia.
The average age of the sample was 79.0 years ( $\mathrm{SD}=6.2$ years), $35.5 \%$ were female and $42.2 \%$ had an annual household income of USD 12,000 or less, which equated to the Australia aged pension in 1992 [25]. The majority of participants of the sample received no support $(65.2 \% ; n=886) ; 16.9 \%(n=229)$ received support from informal sources only; $4.1 \%(n=56)$ from formal sources only and $13.8 \%(\mathrm{n}=188)$ from both informal and formal sources.

Descriptive statistics of study variables by support type are presented in table 1. Compared to recipients of support, those receiving no support were younger, more likely to be male, married and had better mental and physical health.

\section{Measures}

Sociodemographic variables comprised gender, age, income, education and marital status. Gender was a binary variable $(0=$ female, $1=$ male) and age was a continuous variable with a range from 70 to 103. Income was assessed as annual gross household income. Due to positive skewness, the variable was dichotomized into the categories coded as ' $0=$ USD 12,000 or less' and ' $1=$ more than USD 12,000'. Education was defined as the age at which the respondent left school and was also dichotomized due to positive skewness. The two resulting categories were ' $0=$ less than 14 years' and ' $1=14$ years or over'. Marital status was found to be highly correlated with the variable 'living alone' $(r=0.853)$. As the focus of the study was on the association between received support and depressive symptoms, the 'living alone' variable was retained for 
the current analyses. Living alone was coded as ' $0=$ living alone' versus ' 1 = not living alone'.

Cognitive status was screened using a 21-item version of the Mini-Mental Status Examination (MMSE) [26]. The MMSE has been widely used in research to assess for cognitive impairment in older adults as has this shorter version [e.g. 6, 27]. Items included assessed orientation, registration, attention, calculation, and recall [28] with a total score of 16 and under considered indicative of possible cognitive impairment [27]. A binary variable representing those without cognitive impairment (coded as 0 ) and those with possible cognitive impairment (coded as 1) was included in the analyses.

Number of chronic conditions and number of medications has been used in numerous studies to adjust for pathology [e.g. 29]. Number of chronic conditions was assessed by the sum of ten common conditions (arthritis, cancer excluding non-melanocytic skin cancer, chronic bronchitis or emphysema, diabetes, fractured hip, heart attack, heart condition, hypertension, myocardial infarction or osteoporosis) that the participant reported having ever experienced [25]. Number of medications was measured as the total number of prescribed medications (maximum of five) that participants reported that they had taken, or were supposed to have taken, in the last 2 weeks. This list excluded aspirin, headache pills, laxatives, cold medicine and cough medicine.

Informal social networks: social networks variables consisted of ten indicator items drawn from the Established Populations for Epidemiologic Studies of the Elderly Program and assessed (1) the extent of ties and (2) the morphology of these relationships across kin (children and relatives) and non-kin (friends) relationships. These items have previously been described in detailed elsewhere [30]. In line with previous studies, a three-factor first-order confirmatory factor analyses was undertaken to derive three-factor scores: social networks with (1) children, (2) relatives and (3) friends, which were standardized to allow comparison of their relative contribution. Adequate model-fit $\left(\chi^{2} /\right.$ d.f. $=4.37$; Root Mean Square Error of Approximation (RMSEA) = 0.041; Tucker-Lewis Index $(\mathrm{TLI})=0.982$ and Comparative Fit Index $(\mathrm{CFI})=0.989)$ and composite reliabilities were demonstrated $(\alpha=$ $0.69-0.87)[31]$.

\section{Independent Variables}

Type of support was operationalized as the source of support, or the source combination, of help received for difficulties experienced with instrumental activities of daily living (IADL) and activities of daily living (ADL) in accordance with existing research [e.g. 22, 32]. IADL items consisted of ten items that included housework, preparation of own meals, managing finances and shopping for groceries. ADL questions included eight items that pertained to more everyday activities such as bathing, dressing, eating and using the toilet [33]. Respondents were asked whether they had any difficulties with any of these IADL or ADL, and whether they had received any help regarding these activities apart from when they were in a hospital or nursing home. Respondents who received assistance from a person were also asked whether this was from an informal source (i.e. relative or friend) or from a formal source (i.e. care organization). These responses were collated to form four support types: (a) no support received, (b) informal support received only, (c) formal only, and (d) both informal and formal support received [22].
Functional disability was assessed using a measure of functional limitations (restrictions in performing fundamental physical and mental actions; [1]) to avoid confoundment with the type of support measure. A weighted composite was derived through a one-factor confirmatory factor analysis of five Nagi items [34], which asked respondents whether they had any difficulty with a range of motions including (a) pushing or pulling large objects; (b) stooping or crouching or kneeling; (c) lifting or carrying 10 pounds; (d) reaching or extending arms, or (e) writing or handling or fingering small objects. The response scale was a fivepoint Likert scale that ranged from ' $1=$ no difficulty at all' to ' $5=$ just unable to do it'. Results from the confirmatory factor analysis demonstrated adequate model-fit $\left(\chi^{2} /\right.$ d.f. $=1.40$; RMSEA $[90 \%$ $\mathrm{CI}]=0.014$ [0.00-0.049]; TLI $=0.999$ and CFI $=1.00)$. Measurement invariance using a hierarchy of increasingly stringent constraints was also assessed across the four support groups [35]. The assumption of weak $\left(\chi^{2} /\right.$ d.f. $=5.47$; RMSEA $[90 \% \mathrm{CI}]=0.048$ $[0.039-0.057]$; TLI $=0.948$ and $\mathrm{CFI}=0.974)$ and strong factorial invariance were satisfied $\left(\chi^{2} /\right.$ d.f. $=8.18$; RMSEA $[90 \% \mathrm{CI}]=0.061$ [0.053-0.069]; TLI $=0.917$ and CFI $=0.952)$ but not strict factorial invariance $\left(\chi^{2} /\right.$ d.f. $=21.32$; RMSEA $[90 \% \mathrm{CI}]=0.102[0.096-$ $0.107]$; TLI $=0.765$ and CFI $=0.724)$. Estimates assuming strong factorial invariance were utilized. Composite reliabilities were adequate across the four support groups $(\alpha=0.72-0.83)$ [31]. The range of functional limitations across the four support groups was: 0 to 16.12 (no support); 0 to 16.12 (informal support only); 0 to 14.42 (formal support only), and 0 to 16.12 (both informal and formal support).

\section{Dependent Variables}

Depressive symptoms were assessed using the Centre for Epidemiological Studies Depression Scale (CES-D), which was developed to measure depressive symptoms in community-dwelling adults [36]. The CES-D has been shown to have good internal reliability in this sample $(\alpha=0.85)$ [27] and construct and criterion validity amongst older people [37, 38]. It consists of 20 questions that ask participants how they felt in the past week on a four-point Likert Scale that ranged from ' $0=$ rarely or none of the time' to ' 3 = most or all of the time'. Adequate reliability was demonstrated $(\alpha=0.84)$. To reduce positive skewness, we undertook a logarithmic transformation of the total scale scores.

\section{Statistical Analyses}

Hierarchical multiple regression analyses were used to assess the relationships among functional limitations, type of support received and depressive symptoms. Type of support was dummy coded into three variables with no support received as the reference group. In Model 1, covariates were included. Following this, functional limitations (Model 2) and type of support received (Model 3) were added as main effects. In Model 4, the two-way interactions between type of support received and functional limitations were included to examine the stress-buffering effect of type of support on the relationship between functional limitations and depressive symptoms. Finally, the three-way interactions between type of support, functional limitations and age were added to assess whether the strength of the stress-buffering properties of support increased with age. Continuous variables in all interactions were mean centered to avoid colinearity [39]. Missing data were imputed using the expectation maximization algorithm in SPSS 15.0. 


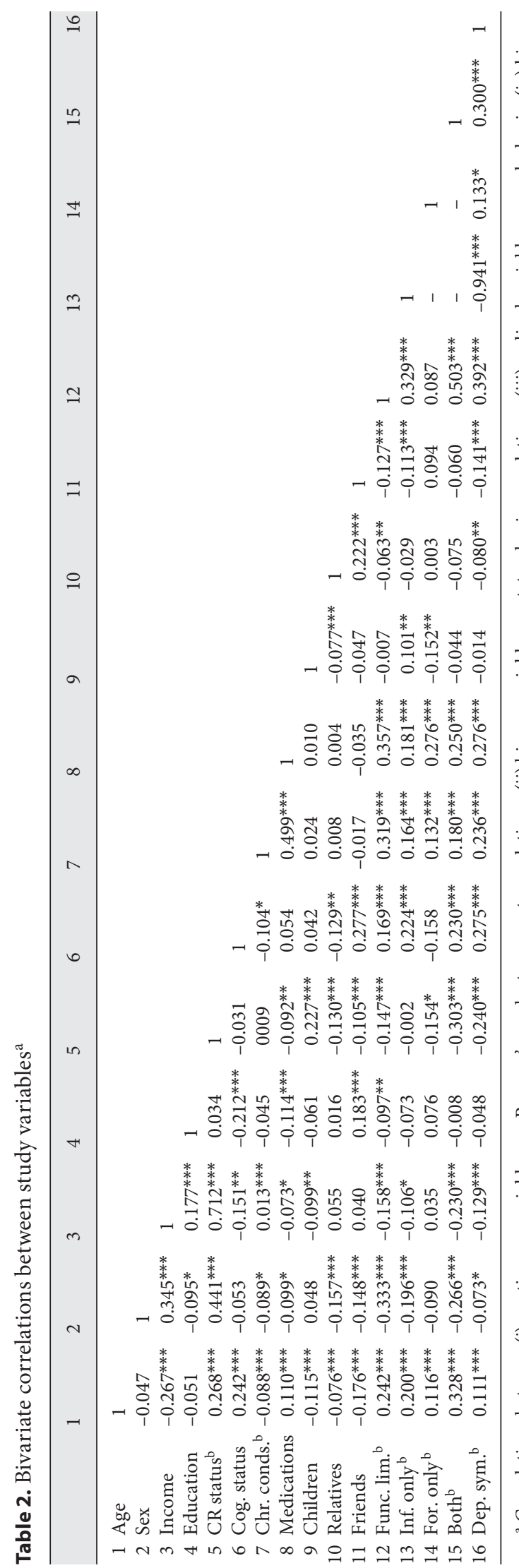

\section{Results}

Bivariate correlations between all variables are shown in table 2. Table 3 presents the results from the hierarchical multiple regression analyses, which examined the direct and interactive relationships among functional limitations, type of support received and depressive symptoms.

\section{Direct Effects of Covariates, Functional Limitations and Type of Support on Depressive Symptoms}

Model 1 showed that none of the sociodemographic variables (age, sex, income and education) were significant predictors of depressive symptoms. By Model 2, sex was a significant predictor with males having greater depressive symptoms. Living alone, having a cognitive impairment, greater number of chronic conditions and medications were all associated with greater depressive symptoms. In relation to social networks, stronger networks with friends was associated with fewer depressive symptoms while a trend suggested that stronger social networks with relatives was also related to lower CES-D scores. Social networks with children was not a significant predictor.

Results from Model 2 showed that the measure of functional limitations was a strong and significant predictor of depressive symptoms contributing an additional $7.9 \%$ of explained variance in depressive symptoms. In Model 3, type of support received was included as direct effect. Results show that these variables contributed minimal variance and were not significant predictors.

\section{Interactive Relationships between Type of Support}

Received, Disability and Depressive Symptoms

Model 4 showed significant interactive effects of (1) informal support only $(b=-0.027, \mathrm{p}<0.10)$ and $(2)$ receipt of both informal and formal support $(b=-0.041$, $\mathrm{p}<0.01)$ on the relationship between functional limitations and depressive symptoms. Analysis of simple slopes revealed that the relationship between functional limitations and depressive symptoms for those receiving (1) informal support only ( $b=0.025,95 \%$ CI [0.014, 0.035], $\mathrm{p}<$ $0.001)$ and (2) both informal and formal support $(b=$ $0.018,95 \%$ CI [0.008, 0.029], $\mathrm{p}<0.001$ ) was weaker as compared to those receiving no support, however significant relationships remained. Figure 1 graphically depicts the stress-buffer effects using predicted scores adjusted for all covariates.

The significant interaction between receipt of both informal and formal support and functional limitations on 


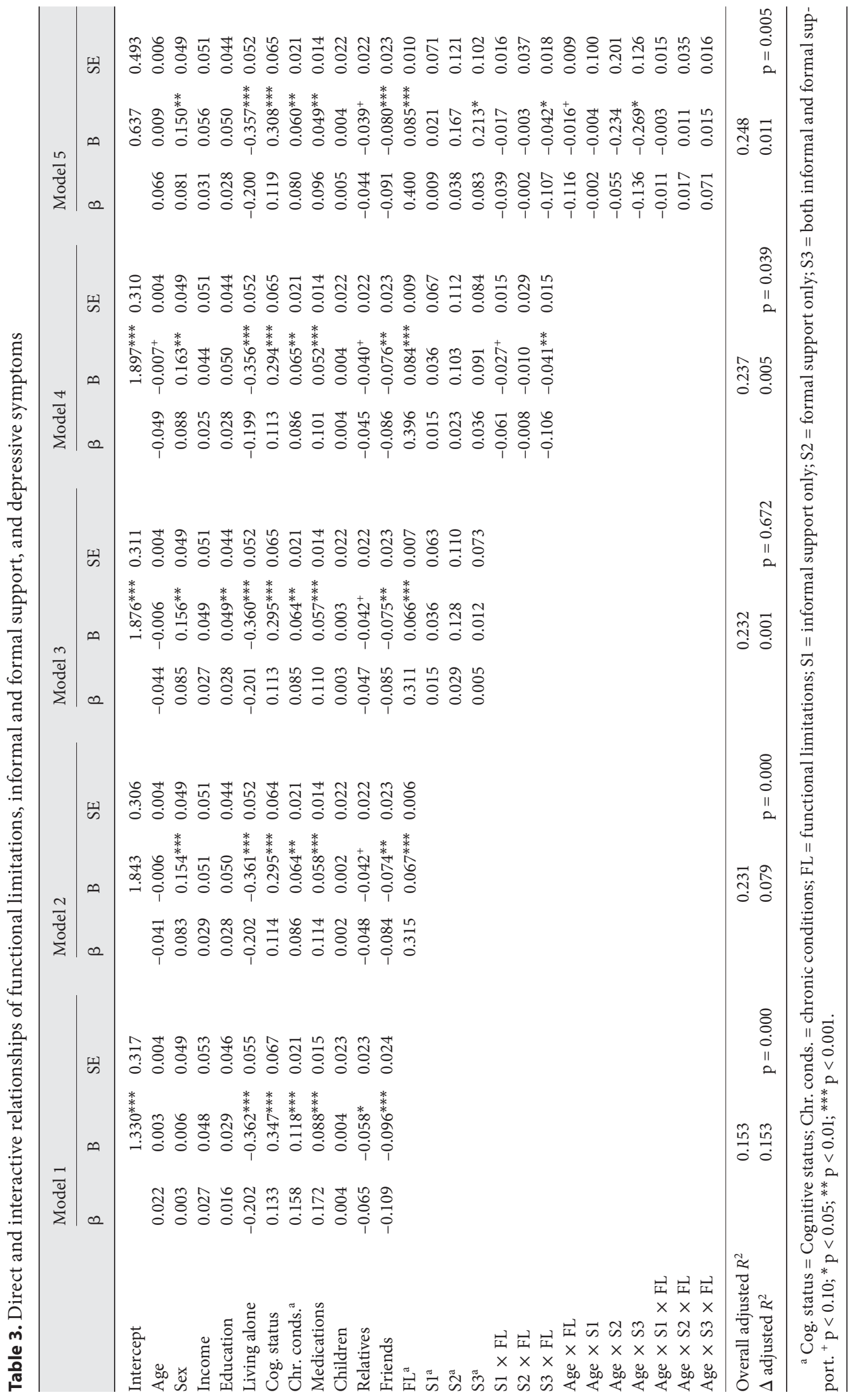




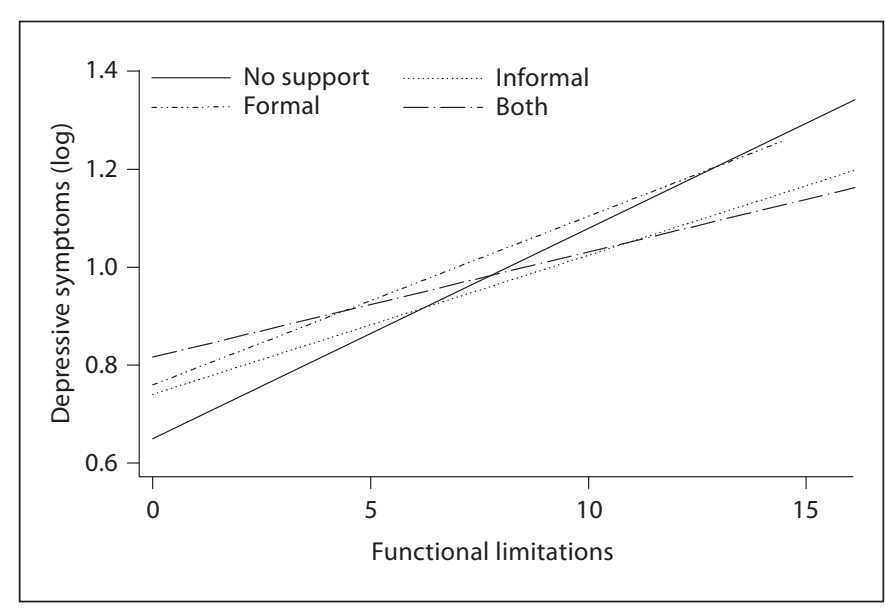

Fig. 1. Moderating effect of different support types on the relationship between functional limitations and depressive symptoms using predicted scores.

depressive symptoms also affected the interpretation of the direct relationship between receiving this support type and depressive symptoms. Specifically, analysis of the simple slopes revealed that for individuals with no functional limitations, receipt of both informal and formal support was not associated with depressive symptoms $(b=0.040, \mathrm{p}>0.05)$. In contrast, for individuals with 1 SD above average functional limitations, receipt of both informal and formal support was related to lower CES-D scores $(b=-0.109, \mathrm{p}<0.05)$.

Model 5 showed that the three-way interactions between age, functional limitations and type of support were not significant indicating that the interaction effect of type of support received by functional limitations did not vary by age. These three-way interactions in Model 5 and the formal support only by functional limitations interaction in Model 4 were also tested at individually in each model to maximize statistical power. All these interactions remained non-significant.

\section{Discussion}

This study contributes to the literature by examining whether enacted instrumental support received from formal sources, either in isolation or in addition to informal support, protects older adults' psychological health against the harmful effects of disability. In addition, the hypothesis that the stress-buffer effect strengthens with increasing age in later life was assessed.
Our results suggest that receiving practical assistance for help with daily activities from both informal and formal sources assists older adults to cope more effectively with the chronic stressor disability relative to those receiving no assistance. Results also showed that receipt of informal support in isolation conferred a protective effect. Together, these results emphasize the importance of enacted support received from informal sources in later life. Informal carers provide the majority of support to community-dwelling older adults. In economic terms, this voluntary contribution has been estimated to be worth close to USD 30.5 billion in Australia [40] and USD 300 billion annually in the United States [41]. Given this, the present findings serve to further emphasize the pivotal role that informal carers play in fostering psychological health amongst community-dwelling older adults.

Another possible interpretation of the significant moderating effect of receiving both informal and formal support is that it is the combination of support from these two sources that confers the protective effect on the relationship between disability and depressive symptoms. Research that suggests recipients of both informal and formal support require assistance from both these sources to have their physical health needs met supports this proposal. For instance, studies show that individuals receiving both informal and formal support have greater physical needs than individuals relying solely on informal support [21, 42]. In addition, empirical studies indicate that older adults often draw upon formal support when their physical needs exceed the capabilities of their existing informal support $[43,44]$. These studies suggest that recipients of both informal and formal support are individuals with greater disability who require assistance from both these sources to satisfy their physical health needs. Further support for the proposal that it may be the combination of informal and formal support, rather than the receipt of informal support, that provides a protective effect comes from the task-specificity model [45]. This model asserts that formal and informal carers are suited to assisting with different tasks. Due to the structure of their organizations, formal providers are preferred for technical and routine tasks that may be carried out at scheduled times. Conversely, informal carers are favorable for non-uniform and diffuse tasks that do not require special knowledge or skills. Accordingly, it may be the combination of informal and formal support that provides the stress-buffering influence.

In contrast, enacted support received from formal sources only did not ameliorate the harmful effects of disability on depressive symptoms, suggesting that formal 
support in isolation may not be sufficient to offset the psychological impact of physical health decline. There may be several explanations for this unexpected result. First, according to the task-specificity model, formal support is unable to adequately assist with the diffuse and unstructured daily activities needs, which may be best met by informal carers [45]. However, research that has assessed the task-specificity model provides minimal evidence for the strict division between informal and formal support [46]. Alternatively, the null result may be due to limited statistical power due to the relatively smaller size of this subgroup. However, examination of the size of the regression coefficient for the interaction term reveals that this proposal is unlikely. Another possible explanation for the null finding regarding the receipt of formal support in isolation is that its nature or quality is insufficient to protect psychological health. Research shows that the quality rather than quantity of social support is a more pertinent predictor of depressive symptoms [47]. In addition, some research suggests that funding constraints and difficulties in attracting, retaining and training staff in the home care industry make it difficult for service providers to ensure high quality care $[3$, 48]. Alternatively, there may be unmeasured factors such as attitudes towards the receipt of support that distinguish those who receive formal support only from individuals in other support groups. However, we did attempt to adjust for such characteristics by including factors that previous research has found to discriminate individuals receiving different support combinations. Finally, it may be the lack of informal support that underlies the lack of protective effective associated with relying solely on formal support. Due to their personal relationship with the care recipient, informal carers are also likely sources of emotional support, which is associated with weaker associations between stress and psychological health in later life [20]. Given that an increasing number of older adults will be relying on formal services in the coming decades, it is important that we continue to assess the relationship between the quality of formal care provided and psychological health.

The significant interaction between receipt of both informal and formal support and disability on depressive symptoms also affects the interpretation of the relationship between receiving this support type and depressive symptoms. Specifically, the results indicate that receipt of this support type was associated with fewer depressive symptoms but only for individuals with above average functional limitations. For individuals with no functional limitations, the receipt of both informal and formal support was not associated with depressive symptoms. These findings are consistent with research that indicates support is not necessarily beneficial to psychological health, especially when it is not required [49]. With regard to disability outcomes, several studies also show that receipt of instrumental support is associated with a higher risk of disability onset in later life [50,51]. Together, these findings highlight the complexity in the relationships between disability, instrumental support and depressive symptoms, and caution us against assuming that the provision of any support is beneficial to the care recipient.

The present study found no support for the view that the stress-buffering effect of enacted support is stronger in the later years of older adulthood [4]. Several explanations for this null result are possible. First, only two studies to date have empirically examined this hypothesis and both of these studies have focused upon the stress-buffering qualities of emotional enacted support $[4,20]$. In comparison, the present study investigated the moderating impact of instrumental enacted support. This variation is important as one of the primary theoretical reasons driving the stress-buffer age variation hypothesis is socioemotional selectivity theory [19]. It may be emotional rather than instrumental enacted support that becomes increasingly pertinent in late life as this coping resource addresses the primary goal of maintaining a sense of connectedness and personal meaning $[19,52]$. Further research is required to assess this hypothesis. Alternatively, the nonsignificant three-way interactions may have been a result of insufficient statistical power, which is a common limitation amongst studies examining moderating effects [53]. Third, the null result may have been due to sample selection bias. Unwell participants are less likely to enroll in longitudinal studies, especially studies that involve relatively long time frames [54]. Given this, the older participants at Wave 1 in the ALSA are likely to have been healthier, and therefore possess a broader time perspective, than their same-aged peers in the general population. Such a sample bias would have led to an underestimation of the stress-buffer age variation effect in this study.

Results for social networks are consistent with past research that suggests discretionary relationships (i.e., friends) as opposed to obligatory relationships (i.e., with children) may be particularly pertinent to health outcomes including psychological health [55], physical health [51] and mortality risk [25]. Together, this research highlights the importance of developing and maintaining programs and initiatives that foster older adult's informal social relationships. 
Several limitations to this study should be noted. First, tests of the stress-buffer age variation hypothesis may have been limited by low statistical power. Numerous factors affect power including the relative size of the subgroups of categorical moderating variables [53]. Given this, future studies with larger and more comparably sized subgroups may wish to re-examine this hypothesis. Second, as mentioned above, the stress-buffer age variation effect may have been affected by sample bias. Longitudinal research that enables the influence of chronological aging, separate from sampling effects, is required to more robustly assess this hypothesis. Third, as this study was cross-sectional, the causal direction of the proposed relationships cannot be empirically determined. Fourth, the impact of the quality of support provided was unable to be examined. Past research has demonstrated that the quality of support affects the relationship between support and psychological health. Further, investigators have warned against assuming support is always desired and provided in a warm and caring manner [49]. Hence, the buffering effect of type of support is likely to be confounded with the quality of the support provided. This study drew a boundary between informal and formal support. However, this distinction is theoretical and it is important to note that in practice there is often a blurring of the informal-formal dichotomy [56]. Finally, the nature and effect of formal support is inextricably confounded with nature of community care policies [22]. Cross-national studies will enable varying home care practices and policies to be compared, thereby improving approaches to fostering healthy aging amongst older adults with disabilities.

Our findings suggest that formal support may play an important role in complementing existing informal support to help maintain quality of life for older adults facing increasing physical health decline. In addition, the central role of informal carers is further emphasized. Although receiving formal support in isolation has the potential to act as an important coping resource in later life, the findings suggest that the nature and quality of formal services need to be reviewed to enable these services to reach their optimal effectiveness. Given that the utilization of formal services is expected to continue to grow in the coming decades in many nations due to demographic changes and the overwhelming preference amongst older adults to age-in-place, this is an important goal.

\section{Acknowledgements}

This article is based upon the Australian Longitudinal Study of Ageing, which is research funded by the South Australian Health Commission, the Australian Rotary Health Research Funds, The US National Institute of Health (grant No. AG 0852302), and the National Health and Medical Research Council (NHMRC) project grants (No. 229936 and No. 179839). The authors gratefully acknowledge the study participants and the staff and researchers at the Centre for Ageing Studies, Flinders University, Adelaide, Australia, for collecting and managing the data.

\section{References}

1 Verbrugge LM, Jette AM: The disablement process. Soc Sci Med 1994;38:1-14.

$\checkmark 2$ Barrera M: Distinctions between social support concepts, measures, and models. Am J Community Psychol 1986;14:413-445.

-3 Montgomery RJ, Holley L, Deichert J, Kosloski K: A profile of home-care workers from 2000 census: how it changes what we know. Gerontologist 2005;45:593-600.

4 Krause NM: Exploring age differences in the stress-buffering function of social support. Psychol Aging 2005;20:714-717.

5 Ormel J, Rijsdijk F, Sullivan M, van Sonderen E, Kempen GIJM: Temporal and reciprocal relationship between IADL/ADL disability and depressive symptoms in late life. $J$ Gerontol B Psychol Sci Soc Sci 2002;57:P338P347.
Anstey KJ, von Sanden C, Sargent-Cox K, Luszcz MA: Prevalence and risk factors for depression in a longitudinal, populationbased study including individuals in the community and residential care. Am J Geriatr Psychiatry 2007;15:497-505.

7 Gayman MD, Turner RJ, Cui M: Physical limitations and depressive symptoms: exploring the nature of the association. J Geront Soc Sci 2008;63B:S219-S228.

$\checkmark 8$ Ganguli M, Hiroko DH, Mulsant BH: Rates and predictors of mortality in an aging, rural, community-based cohort. Arch Gen Psychiatry 2002;59:1046-1052.

-9 Newsom JT, Schulz R: Social support as a mediator in the relation between functional status and quality of life in older adults. Psychol Aging 1996;11:34-44.

10 Taylor MG, Lynch SM: Trajectories of impairment, social support, and depressive symptoms in later life. J Gerontol Soc Sci 2004;59B:S238-S246.
1 Pearlin LI, Menaghan EG, Lieberman MA, Mullan JT: The stress process. J Health Soc Behav 1981;22:337-356.

12 Hebert R, Brayne C, Spiegelhalter D: Incidence of functional decline and improvement in a community-dwelling, very elderly population. Am J Epidemiol 1997; 145:935944.

13 Cohen S: Social relationships and health. Am Psychol 2004;59:676-684

14 Jang Y, Haley WE, Small BJ, Mortimer JA: The role of mastery and social resources in the associations between disability and depression in later life. Gerontologist 2002;42: 807-813.

15 Penninx BW, van Tilburg T, Boeke A, Deeg DJ, Kriegsman DM, van Eijk JT: Effects of social support and personal coping resources on depressive symptoms: different for various chronic diseases? Health Psychol 1998; 17:551-558. 
16 Penninx BW, van Tilburg T, Deeg DJ, Kriegsman DM, Boeke AJ, van Eijk JT: Direct and buffer effects of social support and personal coping resources in individuals with arthritis. Soc Sci Med 1997;44:393-402.

-17 Cohen S, Wills TA: Stress, social support, and the buffering hypothesis. Psychol Bull 1985;98:310-357.

18 Lazarus RS, DeLongis A: Psychological stress and coping in aging. Am Psychol 1983; 38:245-254.

19 Carstensen LL, Isaacowitz DM, Charles ST: Taking time seriously: a theory of socioemotional selectivity theory. Am Psychol 1999; 54:165-181.

20 Krause NM: Lifetime trauma, emotional support, and life satisfaction among older adults. Gerontologist 2004;44:615-623.

21 Kelman HR, Thomas C, Tanaka JS: Longitudinal patterns of formal and informal social support in an urban elderly population. Soc Sci Med 1994;38:905-914.

22 Davey A, Femia EE, Zarit SH, Shea DG, Sundstrom G, Berg S, Smyer MA, Savla J: Life on the edge: patterns of formal and informal help to older adults in the United States and Sweden. J Geront Soc Sci 2005;60B:S281S288.

23 Andrews G, Clark M, Luszcz MA: Successful aging in the Australian Longitudinal Study of Aging: applying the MacArthur model cross-nationally. J Soc Issues 2002;58:479765.

24 Birkett NJ: Computer-aided personal interviewing: a new technique for data collection in epidemiological surveys. Am J Epidemiol 1988;127:684-690.

25 Giles LC, Glonek GF, Luszcz MA, Andrews GR: Effect of social networks on 10 year survival in very old Australians: the Australian Longitudinal Study of Ageing. J Epidemiol Community Health 2005;59:574-579.

-26 Folstein MF, Robins LN, Helzer JE: The Mini-Mental State Examination. Arch Gen Psychiatry 1983;40:812.

27 Luszcz MA, Bryan J, Kent P: Predicting episodic memory performance of very old men and women: contributions from age, depression, activity, cognitive ability, and speed. Psychol Aging 1997;12:340-351.

28 Teng EL, Chui HC, Schneider LS, Metzger LE: Alzheimer's dementia: performance on the Mini-Mental State Examination. J Consult Clin Psychol 1987;55:96-100.
29 Anstey KJ, Luszcz MA, Giles LC, Andrews GR: Demographic, health, cognitive, and sensory variables as predictors of mortality in very older adults. Psychol Aging 2001;16: 3-11.

30 Giles LC, Metcalf PA, Anderson CS, Andrews GR: Social networks among older Australians: a validation of Glass's model. J Cancer Epidemiol Prev 2002;7:195-204.

- 31 Werts CE, Rock DR, Linn RL, Joreskog KG: A general method of estimating the reliability of a composite. Educ Psychol Meas 1978; 38:933-938.

32 Shea D, Davey A, Femia EE, Zarit SH, Sundstrom G, Berg S, Smyer MA: Exploring assistance in Sweden and the United States. Gerontologist 2003;43:712-721.

33 Fillenbaum GG: Multidimensional Functional Assessment of Older Adults: the Duke Older Americans Resources and Services Procedures. Hillsdale/NJ, Erlbaum, 1988.

34 Nagi S: An epidemiology of disability among adults in the United States. Milbank Q 1976; 54:439-468.

35 Meredith W: Measurement invariance, factor analysis, and factorial invariance. Psychometrika 1993;58:525-543.

36 Radloff LS: The CES-D scale: a self-report depression scale for research in the general population. Appl Psychol Meas 1977;1:385401.

37 Lewinsohn PM, Seeley JR, Roberts RE, Allen NB: Center for Epidemiologic Studies Depression Scale (CES-D) as a screening instrument for depression among communityresiding adults. Psychol Aging 1997;12: 227-287.

38 Beekman AT, Deeg D, Van Limbeek J, Braam $A$, et al: Criterion validity of the center for Epidemiologic Studies Depression Scale (CES-D): results from a community-based sample of older subjects in the Netherlands. Psychol Med 1997;27:231-235.

39 Cohen J, Cohen P, West SG, Aiken LS: Applied Multiple Regression/Correlation Analysis for the Behavioral Sciences, ed 3. Mahwah/NJ, Erlbaum, 2003.

40 Report by Access Economics for Carers Australia: The Economic Value of Informal Care. Canberra, Access Economics, 2005.

41 Polivka L: The ethics and politics of caregiving. Gerontologist 2005;45:557-561.

42 Geerlings SW, Pot AM, Twisk JWR, Deeg D: Predicting transitions in the use of informal and professional care by older adults. Ageing Soc 2005;25:111-130.
43 Litwin H, Attias-Donfut C: The inter-relationship between formal and informal care: a study in France and Israel. Ageing Soc 2009;29:71-91.

44 Chappell NL, Blandford A: Informal and formal care: exploring the complementarity. Ageing Soc 1991;11:299-317.

45 Litwak E: Helping the Elderly: the Complementary Roles of Informal Networks and Formal Systems. New York, Guilford Press, 1985.

46 Penning MJ: Receipt of assistance by elderly people: hierarchical selection and task specificity. Gerontologist 1990;30:220-228.

47 Antonucci TC, Akiyama H: Social networks in adult life and a preliminary examination of the convoy model. J Gerontol 1987;42:519527.

48 Thomas M, Woodhouse B, Rees-Mackenzie $\mathrm{J}$ : Use of and satisfaction with community aged care packages in the eastern suburbs of Sydney. Australas J Ageing 2007;26:8-14.

49 Krause NM: Assessing stress-buffering effects: a cautionary note. Psychol Aging 1995; 10:518-526.

50 Avlund K, Lund R, Holstein BE, Due P: Social relations as determinant of onset of disability in aging. Arch Gerontol Geriatr 2004; 38:85-99.

51 Mendes de Leon CF, Glass TA, Beckett LA Seeman TE, Evans DA, Berkman LF: Social networks and disability transitions across eight intervals of yearly data in the New Haven EPESE. J Gerontol Soc Sci 1999; 54B:S162-S172.

52 Mancini AD, Bonanno GA: Marital closeness, functional disability, and adjustment in late life. Psychol Aging 2006;21:600-610.

53 Aguinis H: Statistical power problems with moderated multiple regression in management research. J Manage 1995;21:1141-1158.

54 Morrell CH, Brant LJ, Ferrucci L: Model choice can obscure results in longitudinal studies. J Gerontol Med Sci 2009;64A:215222.

55 Prince MJ, Harwood RH, Thomas A, Mann AH: A prospective population-based cohort study of the effects of disablement and social milieu on the onset and maintenance of latelife depression. The Gospel Oak Project VII. Psychol Med 1998;28:337-350.

56 Porter EJ, Ganong LH, Drew N, Lanes TI: A new typology of home-care helpers. Gerontologist 2004;44:750-759. 